\title{
On lottery sales, jackpot sizes and irrationality: A cautionary note
}

\author{
D.A. Peel ${ }^{*}$ \\ Lancaster University Management School, Lancaster, LA1 4YX UK, United Kingdom
}

\section{A R T I C L E I N F O}

\section{Article history:}

Received 16 November 2009

Received in revised form 12 August 2010

Accepted 20 August 2010

Available online 21 October 2010

JEL classification:

C72

$\mathrm{C} 92$

D80

D84

Keywords:

Lottery sales

Cumulative prospect theory

Jackpot size

Irrationality

\begin{abstract}
A B S T R A C T
Jackpot size has a greater impact than expected price as a determinant of lottery sales suggesting that agents exhibit irrational lotto mania. We demonstrate why this conclusion is problematical by considering the reduced form obtained if agents are described by Cumulative Prospect Theory.
\end{abstract}

(C) 2010 Elsevier B.V. All rights reserved.

\section{Introduction}

Numerous time series studies have been conducted on the determinants of lottery sales in various countries (see e.g. Beenstock and Haitovsky (2001), Cook and Clotfelter (1993), Walker and Young (2001) and Forrest et al. (2002)). The majority of the literature has been concerned to estimate the impact of either jackpot size or the expected price of the lottery on lottery sales. The typical empirical finding, ceteris paribus, has been that lottery sales vary inversely with the expected price of the lottery ticket and positively with the size of the jackpot. However the jackpot size appears to have a much greater statistical impact than expected price. This has given rise to the suggestion that lottery sales exhibit irrational behavior of agents, sometimes called lotto mania.

A feature of the reported studies is that the estimated relationships are not based on models of agents that can both generate gambling at actuarially unfair odds and also explain the preference of many agents for lottery tickets involving a number of prizes. As noted by Walker and Young (2001) some researchers employ the jackpot size as the sole explanatory variable in regression analysis. This raises the questions of why lottery operators do include smaller prizes and why they fail to further decrease the odds of winning an increased jackpot, proportionately, in order to increase profits.

One model that can explain gambling at actuarially unfair odds and also explain the preference of many agents for lottery tickets

\footnotetext{
* Tel.: + 44152465201.

E-mail address: d.peel@lancaster.ac.uk.
}

involving a number of prizes is Cumulative Prospect Theory (CPT) of Tversky and Kahneman (1992). This non-expected utility model embodies probability distortion, and can therefore explain the preference of many agents for multiple prize lottery tickets (see Quiggin (1991)). It has been employed to explain the choices made between safe and risky outcomes in numerous experiments. See e.g. Tversky and Kahneman (1992) and Scott (2006). From a theoretical perspective CPT has been employed to explain, for example, the equity risk premium puzzle (Benartzi and Thaler (1995)). Consequently it appears of interest to determine its implications for the specification and interpretation of the lottery sales reduced form equations estimated in empirical work. That is the purpose of this letter.

In Section 1, we simulate data from the value maximizing stake function of a representative CPT agent gambling on a one prize lottery. We employ this data to run regressions of the type run on multiple prize lotteries and illustrate the misleading nature of the implications that could flow. Given these results we consider the implications for reduced form equations that endeavour to explain sales of multiple prize lotteries. The letter concludes with a brief conclusion.

\section{Section 1: Optimal staking on lotteries in a CPT framework}

Defining reference point utility as zero, expected value in the CPT model, for a gamble with one known outcome, is given by

$E u=w(p) U^{g}(s o)-w(1-p) U^{l}(s)$ 
where $p$ is the objective probability, ois the odds, $s$ is the stake, $U^{g}$ (so) is the value derived from the gain, $U^{l}(s)$ is the disutility derived from the loss and $w^{+}(p)$ and $w^{-}(1-p)$ are the respective weighting functions over gains and losses.

We employ the expo-power function of Saha (1993) as our parametric choice of value function. The expo-power function nests the power value function of Tversky and Kahneman but resolves a number of theoretical and empirical objections to that specification. ${ }^{1}$

Substitution of the expo-power value functions in Eq. (1) gives us

$E u=w^{+}(p)\left(1-e^{-r \alpha(s o)^{n}}\right)-w^{-}(1-p) k\left(1-e^{-\alpha s^{n}}\right)$

where $r, \alpha, n$ and $k$ are positive constants.

With $n \leq 1$ the agent is risk averse over gains and risk loving over losses as hypothesized by Tversky and Kahneman and nests the power value function specification of Tversky and Kahneman as $\alpha \rightarrow 0$ (by L'Hopital's Rule) or the exponential function, $\mathrm{n}=1$.

We assume that the probability weighting function over gains, $w^{+}(p)$, and losses, $w^{-}(1-p)$, are given by the parametric forms assumed by Tversky and Kahneman (1992):

$w^{+}(p)=\frac{p^{\delta}}{\left[p^{\delta}+(1-p)^{\delta}\right]^{\frac{1}{\delta}}}$

and over losses

$w^{-}(1-p)=\frac{(1-p)^{\rho}}{\left[p^{\rho}+(1-p)^{\rho}\right]^{\frac{1}{p}}}$

where $\delta, \rho$ are positive constants. For $\delta, \rho<1$ we have over weighting of low probabilities and underweighting of large probabilities.

Differentiation of Eq. (2) with respect to stake size gives us the optimal stake size, $s$, as

$s=\left[\frac{\ln \frac{w^{+}(p) r o^{n}}{w^{-}(1-p) k}}{\alpha\left(r o^{n}-1\right)}\right]^{\frac{1}{n}}$

The second order condition for a maximum is $r 0^{n}-1>0$.

We define the expected return $(\lambda)$ from a one unit stake gamble on the one prize lottery as

$\lambda=p o-(1-p)$

$1-\mu$

Letting $\mu=1+\lambda$, the expected price of a lottery ticket is $1-(1+\lambda)=$

From Eq. (6) odds, $o$, are given by

$o=\frac{\mu}{p}-1$

The variance of expected return, $\sigma^{2}$, for this one prize lottery is given by

$\sigma^{2}=p(0-\lambda)^{2}+(1-p)(-1-\lambda)^{2}$

\footnotetext{
${ }^{1}$ For example in experiments where agents choose between a 'safer' and 'more risky' gamble many report that risk aversion increases sharply as payoffs are increased. See e.g. Holt and Laury (2002) who employ the expo-power function. Also De Giorgi and Hens (2006) demonstrate that the power value function leads to the nonexistence of equilibria in some applications in portfolio theory. They suggest the exponential function, as an alternative, which is nested by the expo-power function employed above. Peel and Law (2008) provide an application to some other forms of gambling.
}

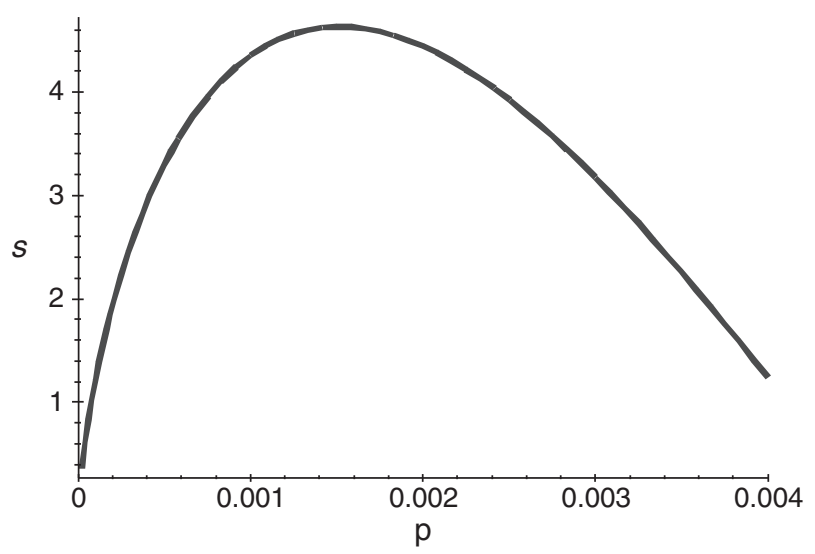

Fig. 1. Optimal stake $\mu=0.5$.

Which from Eqs. (7) and (8) implies that

$p=\frac{\mu^{2}}{\mu^{2}+\sigma^{2}}$

From inspection of the determinants of the optimal stake size (Eq. (5)) we see that it depends, non-linearly, on the objective probability and the odds. Consequently it immediately follows from Eqs. (7) and (9) above that the optimal stake can be written solely as a complicated non linear function of any two of the expected price of a lottery ticket, the variance of expected returns or the odds.

For instance writing $o^{n}$ solely as a function of $\mu$ and $\sigma^{2}$ we obtain the expression $o^{n}=\left[\mu+\frac{\sigma^{2}}{\mu}-1\right]^{n}$. This term appears in the denominator and its logarithm in the numerator of the optimal stake (Eq. (5)). The ratio of the probability weighting functions in Eq. (5) is also a complex function of $\mu$ and $\sigma^{2}$.

To illustrate the implications of the model for stake size we assume that a representative KT agent is described by the following parameters;

$\delta=0.61, \rho=0.69, n=0.88, \alpha=0.0001, r=5$ and $k=11.25$. These parameter values give us near identical outcomes over small stakes and a wide range of odds as in the original power function specification of Tversky and Kahneman (1992). However the bounded value functions imply different behavior over large enough odds.

In Figs. 1 and 2 we plot the optimal stake as a function of objective probability for these parameter values when $\lambda=-0.5$ and -0.55 . We observe from the figures that the optimal stake exhibits a maximum. The maximum reflects the interplay of probability distortion and boundedness of the value function. Essentially as the odds become

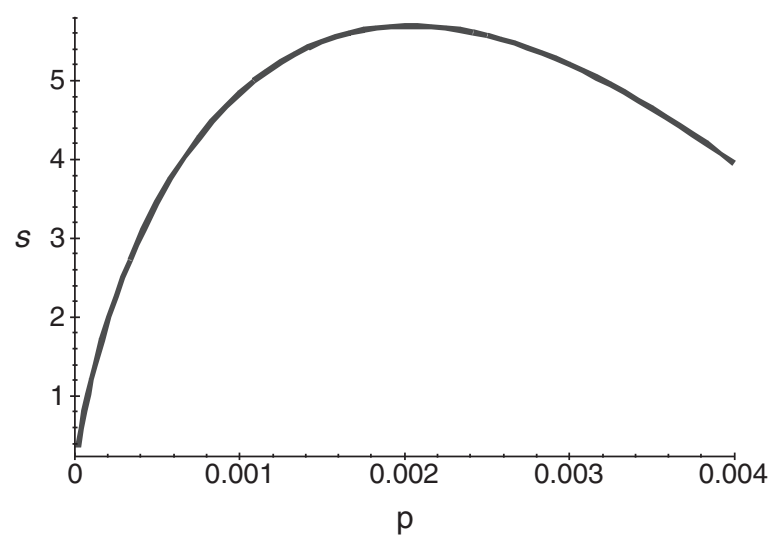

Fig. 2. Optimal stake $\mu=0.55$. 
Table 1

Least squares regressions for simulated data and its determinants.

\begin{tabular}{llllll}
\hline $\begin{array}{l}\text { Dependent } \\
\text { variable }\end{array}$ & Constant & $\log (1-\mu)$ & $\log \sigma^{2}$ & $\log (0)$ & $\bar{R}^{2}$ \\
\hline$s$ & -0.0295 & -0.0215 & -0.0231 & 0.0229 & 0.817 \\
& $(0.0021)^{*}$ & $(0.0015)^{*}$ & $(0.0017)^{*}$ & $(0.0017)^{*}$ & \\
$s$ & -0.0002 & -0.00098 & $-4.4^{*} 10^{-5}$ & & 0.427 \\
& $\left(3^{*} 10^{-5}\right)^{*}$ & $\left(3.1^{*} 10^{-5}\right)^{*}$ & $\left(2.23^{*} 10^{-6}\right)^{*}$ & & \\
\hline
\end{tabular}

Number of observations $=1908$. White Heteroskedastic-consistent standard errors in parenthesis.

* Significant at the $1 \%$ of significance.

large enough (lower probabilities) utility satiation, due to the assumption of boundedness, implies a lower optimal stake. As probabilities become larger, (lower odds), the upward distortion of probabilities diminishes and ultimately reverses so that positive subjective expected returns are reduced, ultimately becoming negative, so that stake size ultimately becomes zero.

We employed the parameters above to generate 1708 data points for the optimal stake of an agent. We first assume that the expected return $\lambda$, is equal to -0.5 , using 854 probabilities that lay between $3.4910^{-7}$ and 0.004 and which were uniformly distributed across this range. We then repeated this exercise for the same probability range assuming the expected return is -0.55 . The change to a higher expected return mimics the effect of a roll over in a lottery.

Employing the simulated data we report in Table 1 the results obtained from the regression of the level of the optimal stake on the logarithm of expected price, the logarithm of variance and the logarithm of odds. ${ }^{2}$

All variables are significant at the $1 \%$ probability level. The regression results seem to imply that higher expected price, (lower expected return) or higher variance reduces stake size (sales) whilst a greater jackpot size, (odds), increases sales. The empirical results appear consistent with "lotto mania", an inference that is incorrect in this model.

\section{Section 2. Lottery Tickets with more than one payoff}

We continue to assume that an agent, described by CPT, makes an optimal stake decision when deciding to purchase lottery tickets since the implications are similar if we considered it as a discrete decision, namely to purchase zero, one, two or more tickets. To simplify the analysis, though the implications are quite general, we assume the prizes are known prior to purchase but some change from draw to draw. Consider a lottery with a three variable prizes. The lottery has a structure where balls are drawn sequentially from three urns which contain exactly one thousand numbered balls. In order to proceed to a higher prize you have to obtain a correct ball or number in the previous urn.

The expected value of this lottery under CPT, assuming the agent purchases less tickets than the minimum prize size, $z$, is given by, (see Tversky and Kahneman (1992)).

$$
\begin{aligned}
E u= & w^{+}\left(s p^{3}\right) U(x+1-s)+\left\{w^{+}\left(s p^{3}+s p^{2}(1-p)\right)-w^{+}\left(s p^{2}(1-p)\right)\right\} \\
& \times U(y+1-s) \\
& +\left\{w^{+}\left(s p^{3}+s p^{2}(1-p)+s p(1-p)\right)-w^{+}\left(s p^{3}+s p^{2}(1-p)\right)\right\} \\
& \times U(z+1-s)-w^{-}(1-p s) U(s)
\end{aligned}
$$

where the prizes are such that $x>y>z$ and $p$ is the probability of drawing a number, $\frac{1}{1000}$ in this example.

The optimal stake can be obtained from Eq. (10) by differentiation. However it is easy to see that it does not have a closed form solution for the expo-power function or in fact any standard parametric form

\footnotetext{
${ }^{2}$ We repeated the same exercise employing the logarithm of stake and the results have the same implications. (available on request).
}

of the value function. The implicit form of the optimal stake function is, of course, given by

$s=f(p, x, y, z)$

so that optimal stake depends on the probabilities of winning and the different prize sizes.

Naturally if a researcher estimates a lottery equation which is based on Eq. (11) but only includes the jackpot size then clearly the jackpot can be significant, without implying lotto mania, since it is the most important determinant of the reduced form and may be "acting as a proxy for itself". It would appear that previous studies are subject to this criticism since some studies only include the jackpot size.

An alternative approach is to substitute for the prizes $x, y$ and $z$ in Eq. (11) in terms of the central moments, (defined for a one unit stake), expected return, variance and skewness. One could then include the jackpot as an additional regressor. It should be insignificant if the moments included substitute appropriately for the prizes in Eq. (11). ${ }^{3}$ However, as with the one prize lottery considered in the previous section, there is a complex relationship between the prizes, $x, y$ and $z$ and the higher order central moments to which closed form solutions do not exist. ${ }^{4}$ Consequently the moments included my not capture the reduced form as well as inclusion of the jackpot and a few other moments.

\section{Conclusions}

We have illustrated some of the implications of CPT for aggregate studies of lottery sales. We demonstrated why the finding of significance of jackpot size in the reduced form lottery sales equations reported in the literature may not reflect lotto mania. There is an observational equivalence of reduced forms implied by models such as CPT and those that purport to demonstrate lotto mania. As a consequence of the complex mapping from prizes to higher central moments in the lotto game it would appear that the structural interpretation of important policy parameters in previous studies, such as the elasticity of sales with respect to expected price or jackpot size is problematic.

\section{References}

Beenstock, M., Haitovsky, Y., 2001. Lotto mania and other anomalies in the market for lotto. Journal of Economic Psychology 22, 721-744.

Benartzi, S., Thaler, R.H., 1995. Myopic loss aversion and the equity premium puzzle. Quarterly Journal of Economics 110, 73-92.

Cook, P.J., Clotfelter, C.T., 1993. The peculiar scale economies of lotto. American Economics Review 83 (3), 634-643.

Forrest, D., Simmons, R., Chesters, N., 2002. Buying a dream: alternative models of demand for lotto. Economic Inquiry 40 (3), 485-496.

De Giorgi, E., Hens, T., 2006. Making prospect theory fit for finance. Financial Markets and Portfolio Management 20 (3), 339-360.

Holt, C.A., Laury, S.K., 2002. Risk aversion and incentive effects. American Economic Review 97, 1644-1655.

Peel, D.A., Law, D., 2008. A more general non-expected utility model as an explanation of gambling outcomes for individuals and market. Economica 76, 251-263.

Quiggin, J., 1991. On the optimal design of lotteries. Economica 58, 1-16.

Saha, A., 1993. Expo-power utility: a flexible form for absolute and relative risk aversion. American Journal of Agricultural Economics 75, 905-913.

Scott, H.P., 2006. Choosing cumulative prospect theory's functional menagerie. Journal of Risk and Uncertainty 32, 101-130.

Tversky, A., Kahneman, D., 1992. Advances in prospect theory: cumulative representation of uncertainty. Journal of Risk and Uncertainty 5 (4), 297-323.

Walker, I., Young, J., 2001. An economist's guide to lottery design. The Economic Journal $111,700-722$.

${ }^{3}$ This is the implicit approach followed by some researchers, e.g. Beenstock and Haitovsky (2001).

${ }^{4}$ The first three central moments for a unit stake are defined as

$\lambda=p^{3} x+p^{2}(1-p) y+p(1-p) z-(1-p)$

$\sigma^{2}=p^{3}(x-\lambda)^{2}+p^{2}(1-p)(y-\lambda)^{2}+p(1-p)(z-\lambda)^{2}+(1-p)(-1-\lambda)^{2}$

skew $=p^{3}(x-\lambda)^{3}+p^{2}(1-p)(y-\lambda)^{3}+p(1-p)(z-\lambda)^{3}+(1-p)(-1-\lambda)^{3}$

It is not possible to obtain closed form solutions for the prizes $x, y$ and $z$ in terms of $\lambda, \sigma^{2}$ and skew. 